\title{
A feasibility study of total endovascular aortic arch replacement: From stent-graft design to preclinical testing
}

\author{
Jian Yang, MD, PhD, ${ }^{\mathrm{a}}$ Yang Liu, MD, ${ }^{\mathrm{a}}$ Weixun Duan, $\mathrm{MD},{ }^{\mathrm{a}}$ Dinghua Yi, MD, PhD, ${ }^{\mathrm{a}}$ \\ Shiqiang Yu, MD, PhD, ${ }^{\mathrm{a}}$ Rui Ma, MD, ${ }^{\mathrm{b}}$ and Jun Ren, $\mathrm{MD}, \mathrm{PhD}^{\mathrm{c}}$
}

\section{ABSTRACT}

Objective: This study aimed to evaluate the safety and feasibility of total endovascular repair of the aortic arch using a novel designed stent-graft system (Yanger stent-graft system; Lifetech Scientific, Shenzhen, China) in vitro and in a canine model.

Methods: The Yanger stent-graft system is composed of a main stent-graft, branched stent-graft, and a delivery system. In an in vitro aortic arch simulation test bench, the system was tested, and the success rate of stent-graft releasing, anchoring, and positioning was recorded and analyzed. Meanwhile, in vivo implantation of the Yanger stent-graft system was carried out in 16 healthy adult crossbreed dogs. The feasibility of using these stent-grafts was evaluated with aortography, multislice computed tomography, and autopsy 6 months after the procedure.

Results: All stent-graft releasing and positioning were successful for in vitro test. In the canine model, the Yanger stent-graft system was placed successfully in the aortic arch in all 14 dogs with the exception of 2 mortalities. All dogs survived for at least 3 months with normal physiologic signs. Aortography, multislice computed tomography, and animal necropsy revealed good fixation in all cases. The ascending aorta, descending aorta, brachiocephalic trunk, and left subclavian artery were all covered without endoleak. There was no significant obstruction or stenosis in brachiocephalic branches at the 6-month follow-up.

Conclusions: Total endovascular repair of aortic arch with the novel designed Yanger stent-graft system is safe and feasible in preclinical studies. With a better understanding of preclinical knowledge, patient selection criteria and first-inhuman studies will be addressed. (J Thorac Cardiovasc Surg 2016;151:1203-12)

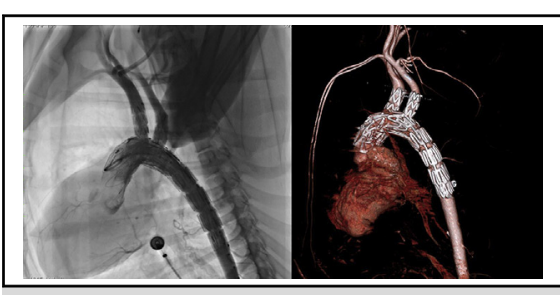

Total endovascular aortic arch replacement is successful in a canine model.

\section{Central Message}

Total endovascular repair of the aortic arch is safe and feasible in preclinical studies.

Perspective

Total endovascular repair of the aortic arch with the novel designed Yanger stent-graft system is safe and feasible in preclinical studies. With increasing preclinical knowledge, patient selection criteria and first-in-human studies will be addressed.

See Editorial Commentary page 1213.

\section{Video clip is available online.}

\footnotetext{
From the Departments of ${ }^{\mathrm{a}}$ Cardiovascular Surgery and ${ }^{\mathrm{b}}$ Anesthesiology, Xijing Hospital, Fourth Military Medical University, Xi'an, China; and ${ }^{\mathrm{c}} \mathrm{S}$ chool of Pharmacy, College of Health Sciences, University of Wyoming, Laramie, Wyo.

This study was supported by funding from the National Natural Science Foundation of China (Grants 81170213, 81470500), the Distinguished Young Scholar Cultivation Project of Xijing Hospital (XJZT14J03), and a grant from the National Key Technology Research and Development Program for the 12th Five-Year Plan (2011BAI11B20).

Received for publication Aug 21, 2015; revisions received Oct 23, 2015; accepted for publication Oct 24, 2015

Address for reprints: Jian Yang, MD, PhD, Department of Cardiovascular Surgery, Xijing Hospital, 710032 Xi'an, China (E-mail: yangjian1212@ hotmail.com). 0022-5223/\$0.00

Copyright (C) 2016 by The American Association for Thoracic Surgery. Published by Elsevier Inc. This is an open access article under the CC BY-NC-ND license (http:// creativecommons.org/licenses/by-nc-nd/4.0/).

http://dx.doi.org/10.1016/j.jtcvs.2015.10.092
}

Aortic diseases (aneurysm and dissection) involving the aortic arch are usually associated with high rates of mortality and morbidity and remain a surgical challenge. ${ }^{1}$ Various approaches have been used to treat aortic disease, including thoracic endovascular aortic repair (TEVAR) via the chimney technique, hybrid debranching techniques, total arch replacement, and frozen elephant trunk operations. ${ }^{2-7}$ These techniques require the use of cardiopulmonary bypass, deep hypothermic circulatory arrest, or sternotomy, which is associated with massive trauma and complications. ${ }^{8-11}$ Total endovascular aortic arch replacement without open surgery is challenging for cardiothoracic surgeons, cardiologists, vascular surgeons, and interventionists. There are a limited number of reports in the literature regarding total endovascular aortic arch replacement. ${ }^{12,13}$ We have developed a new stent-graft system designed for total endovascular arch replacement and have included its preclinical in vitro testing and animal study results in this article. 


\section{Abbreviations and Acronyms \\ $\mathrm{BCT}=$ brachiocephalic trunk \\ LSCA = left subclavian artery \\ MSCT = multislice computed tomography \\ TEVAR $=$ thoracic endovascular aortic repair}

\section{MATERIALS AND METHODS \\ Description of the Stent-Graft System}

The Yanger stent-graft system (Lifetech Scientific, Shenzhen, China) is composed of 3 parts: the primary stent-graft, branched stent-graft, and delivery system. The primary stent-graft is based on the Ankura II stent-graft system (Lifetech Scientific). However, the Yanger stent-graft system consists of a native branch and 1 or 2 in situ fenestrations (Figure 1, $A$ and $B$ ). The diameter of the fenestration and the distance between the fenestration and the branch graft are predetermined and may be modified for individual patients. The use of a polytetrafluoroethylene and nitinol frame affords the primary stent-graft excellent flexibility and allows for modifications to the curvature of the aortic arch. The branched stent-graft is a derby hat-shaped short stent-graft and is composed of a polytetrafluoroethylene and nitinol frame (Figure 1, $C$ and $D$ ). With the use of a delivery system (Figure 1, E and $F$ ), the branched stent-graft may be inserted into the fenestrations of the primary stent-graft. The derby hat-shaped branched stent-graft is designed to reduce the risk of leakage between the fenestrations of the primary stent-graft and the branched stent-graft. The Yanger stent-graft system is designed to mimic the anatomy of a native aortic arch (Figure 1, $G$ and $H$ ). Because animal models possess only 2 super-aortic branches, we have designed a stent-graft with 1 branch and 1 fenestration for the animal study (Figure $1, I$ and $J$ ). Three Yanger stent-graft systems $(24-20-160 \mathrm{~mm}, 28-24-160 \mathrm{~mm}$, and $32-26-160 \mathrm{~mm}$ ) were manufactured for the in vitro testing and the animal studies. Both fenestrations were $15 \mathrm{~mm}$ in diameter, and the distances between the fenestrations and the branch were predetermined as $8 \mathrm{~mm}$. During the animal testing, the length of the covered stent-graft was $40 \mathrm{~mm}$. The diameters of the proximal and distal stents, as well as the branch, fenestrations, and distances between the fenestrations and the branch, may be individualized. The sheath is $23 \mathrm{~F}$ for the $24-\mathrm{mm}$ and $28-\mathrm{mm}$ systems and $24 \mathrm{~F}$ for the $32-\mathrm{mm}$ main stent-graft system. This sheath is a commercially available sheath that is used in Ankura thoracic stent-graft products and is also from LifeTech Scientific.

\section{Release Procedure}

The release procedure of the Yanger stent-graft system was performed as follows: Laparoscopy was performed to expose and cannulate the abdominal aorta. The brachiocephalic trunk (BCT) was punctured and inserted with a $6 \mathrm{~F}$ sheath. Through the sheath, a $6 \mathrm{~F}$ multipurpose catheter with slippery wire was advanced into the abdominal aorta and femoral artery. By making a purse suture of the abdominal aorta, the $6 \mathrm{~F}$ multipurpose catheter was taken out and the branch traction wire of the primary stent-graft was inserted into the catheter and all along the route out of the BCT. A Lunderquist super-stiff guidewire (Cook Inc, Bloomington, Ind) was advanced from the abdominal or femoral artery into the ascending aorta and left ventricle. By using the Lunderquist guidewire, the delivery system and compressed primary stent-graft were advanced into the aortic arch (Figure 2, A). The branch stent-graft of the main graft system is first compressed into a small sheath with a retracting wire inside. By stretching the branch traction wire, simultaneously advancing the primary stent-graft delivery system, and gradually retracting the outer sheath of the delivery system, the compressed branch was guided to the orifice of the BCT and in the desired position (Figure 2, B). By releasing the outer sheath, the distal portion of the stent-graft was opened
(Figure 2,C). By retracting the guidewire through the BCT, the branch of the primary stent-graft was released (Figure 2,D). The safety locker of the delivery system was released, and the front fixation of the primary stent-graft was opened and positioned (Figure 2,E). After full deployment of the primary stent-graft, the delivery sheath was retrieved (Figure 2, F). A super-slippery wire/stiff-wire was introduced into the left subclavian artery (LSCA) through the premade fenestration and advanced toward the orifice of LSCA (Figure 2, G). With the use of the super-stiff guidewire, the delivery system of the derby hat branched stent-graft was advanced into the LSCA (Figure 2, $H$ ). After retrieving the outer sheath and retracting the prebonded thread (Figure 2, $I$ and $J$ ), the branched stent-graft was released (Figure 2, $K$ ). After the retrieval of the delivery system, the procedure was complete (Figure 2, $L$ ).

\section{In Vitro Test}

The Yanger stent-graft system was tested using an in vitro aortic arch simulation test bench (Lifetech Scientific). The simulation test bench was a polyurethane aortic arch model equipped with an ascending aorta, a descending aorta, and 3 brachiocephalic branches, simulating the anatomy of the human aortic arch. By using the simulation test bench, the Yanger stent-graft delivery system was inserted, and the stent-graft was subsequently released (Figure 3, $A-D$ ); the branched stent-graft was subsequently inserted into the fenestrations of the primary stent-graft and released (Figure 3, E-H). The success rate of the stent-graft's release, anchoring, and positioning was recorded and analyzed.

\section{Preparation of Animals}

Sixteen healthy adult crossbreed dogs ( 7 female and 9 male) weighing an average of $28.2 \pm 2.7 \mathrm{~kg}$ were used in this study. Preoperative color echocardiographic examinations were performed, and no abnormalities were found. The skin of the neck, abdomen, and both groins was depilated and sterilized using iodophors. All animals were intubated and ventilated using an animal anesthesia ventilator (Matrx 3000; Midmark Corp, Dayton, Ohio). The animals were anaesthetized via intramuscular injections of ketamine (10 mg/kg of body weight) and vecuronium bromide ( $2 \mathrm{mg}$ ). Propofol $(0.1 \mathrm{mg} / \mathrm{kg} / \mathrm{min})$ was administered intravenously to maintain anesthesia. All procedures were performed with the animals in the dorsal recumbent position. Electrocardiogram readings were monitored throughout the procedure.

\section{Ethics Statement}

All animals received humane care in compliance with the Ministry of Science and Technology of the People's Republic of China Guide for the Care and Use of Laboratory Animals. Both the Institutional Animal Care and Use Committee and the Medical Ethics Committee of Xijing Hospital approved this study and each of its experiments (20120216-4).

\section{Total Endovascular Stent-Graft Implantation in the Aortic Arch}

Catheterization was performed in a specialized animal catheterization laboratory. After experimental animals were fully anesthetized, carotid and femoral/abdominal arteries were mobilized and cannulated. A 6F pigtail catheter was advanced in the ascending aorta, and aortography was performed. After measuring the aortic arch, the pigtail catheter was exchanged for a Lunderquist super-stiff wire. A multipurpose catheter and a 260-mm super slippery wire were introduced into the descending aorta through right carotid artery cannulation, grasped, and pulled through a femoral or an abdominal artery cannulation. By using the multipurpose catheter, the branch wire of the primary stent-graft was introduced. With the use of the Lunderquist super-stiff wire, the entire primary stent-graft system was advanced into the aortic arch. The primary and branch stentgraft systems were deployed as previously described. After the deployment 


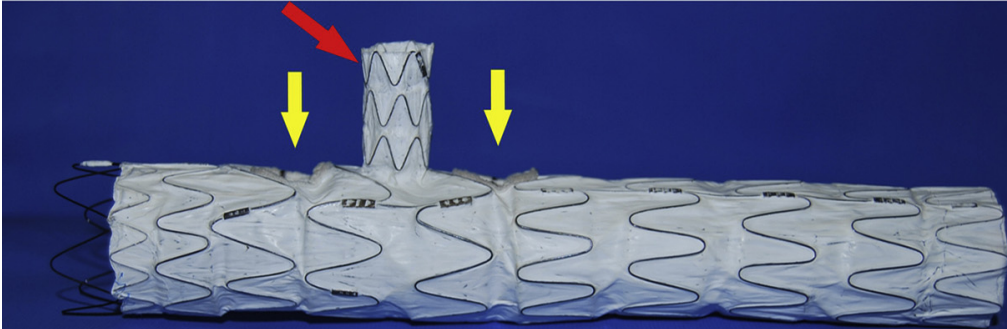

A

C
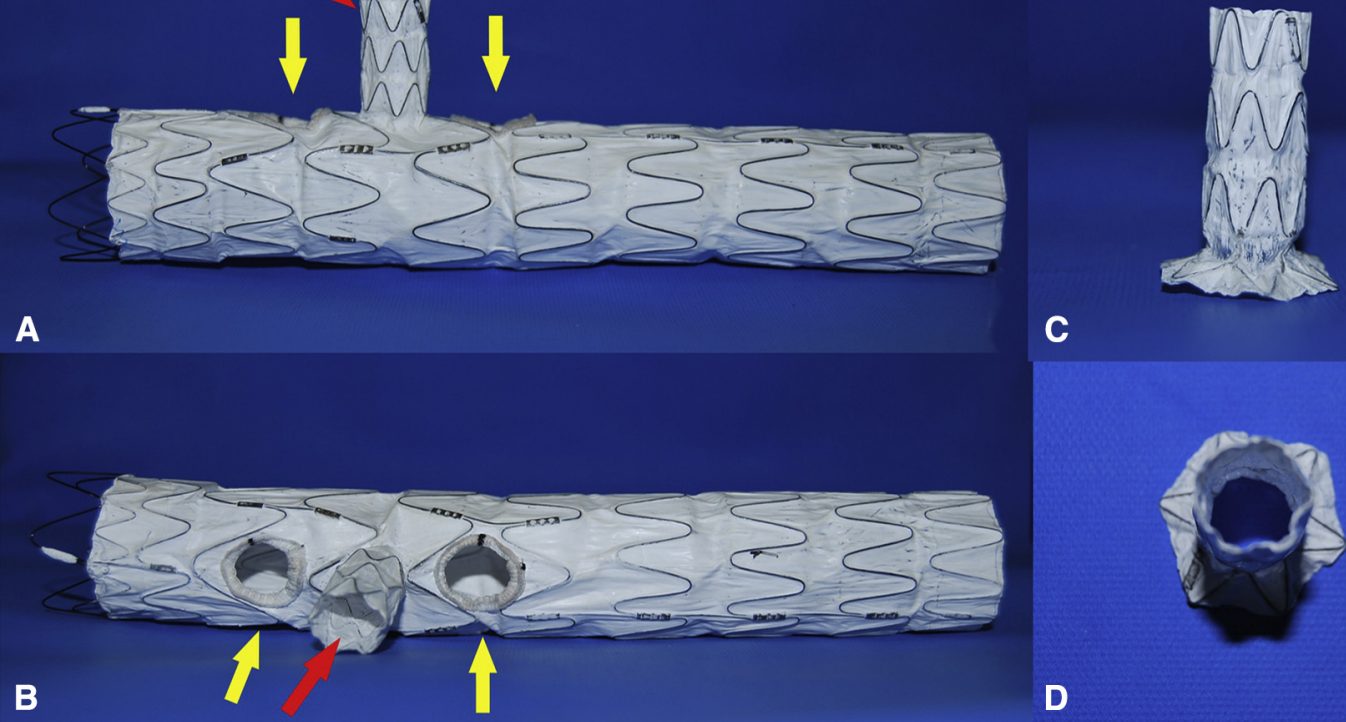

D
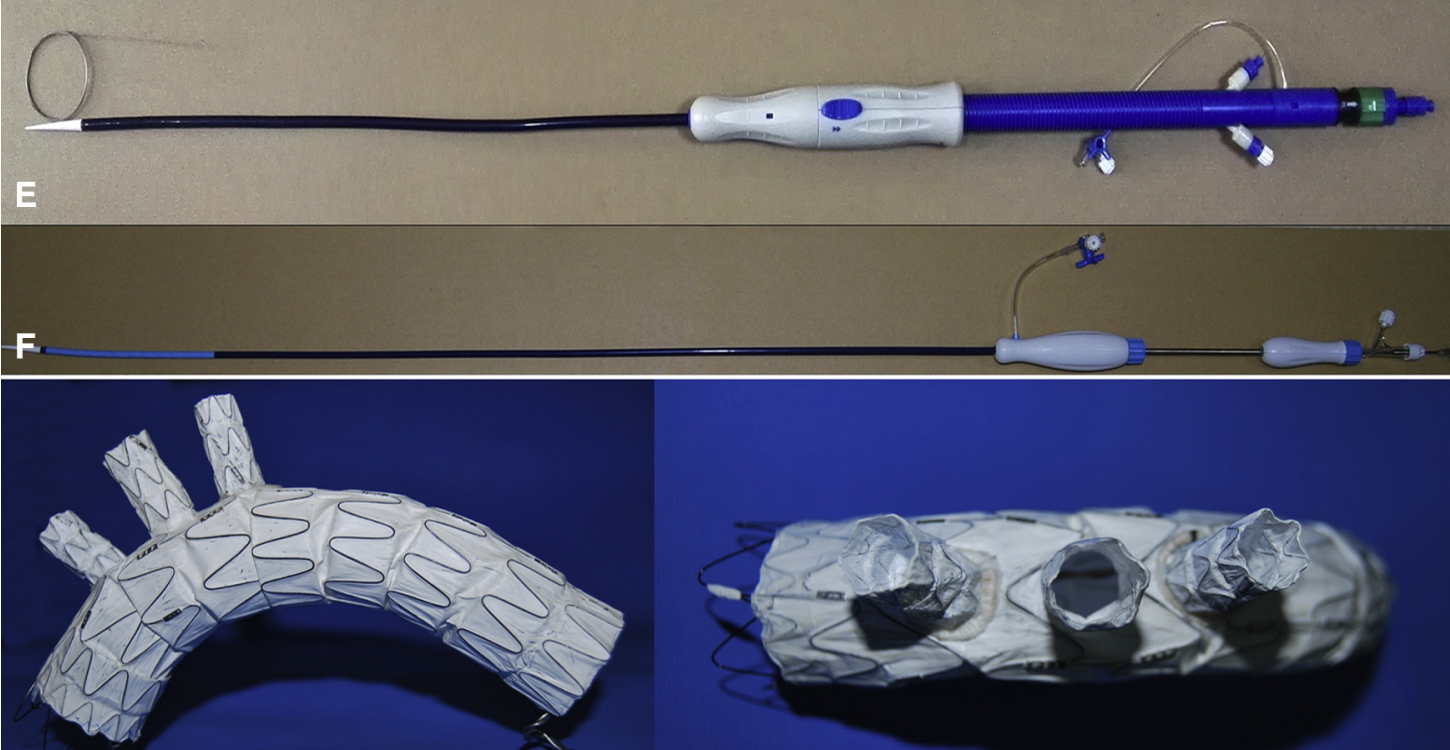

G

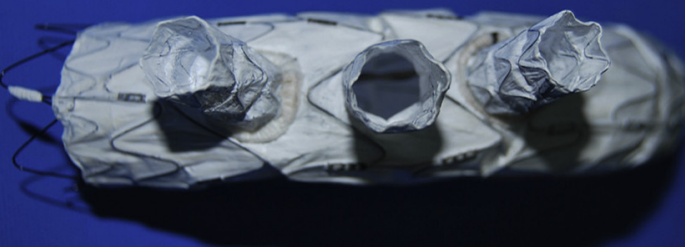

H

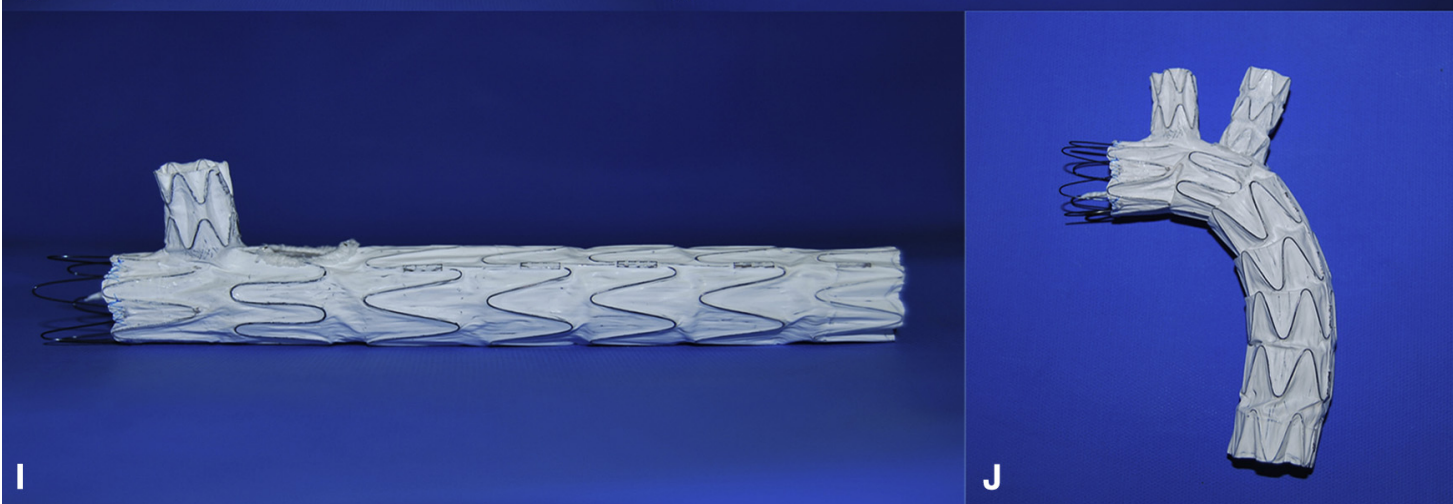

FIGURE 1. Components of the Yanger stent-graft system (Lifetech Scientific, Shenzhen, China). A, Lateral view of the primary stent-graft. B, Frontal view of the primary stent-graft. C, Lateral view of the branched stent-graft. D, Frontal view of the branched stent-graft. E, Delivery system of the primary stent-graft. F, Delivery system of the branched stent-graft. G, Lateral view of the final Yanger stent-graft system. H, Cross-section of the final Yanger stent-graft system. I, Lateral view of primary stent-graft with 1 branch and 1 fenestration designed for the animal study. J, Lateral view of the final Yanger stent-graft system with 2 branches for the animal study. The red arrow denotes the native branch, and the yellow arrow denotes the in situ fenestrations. 

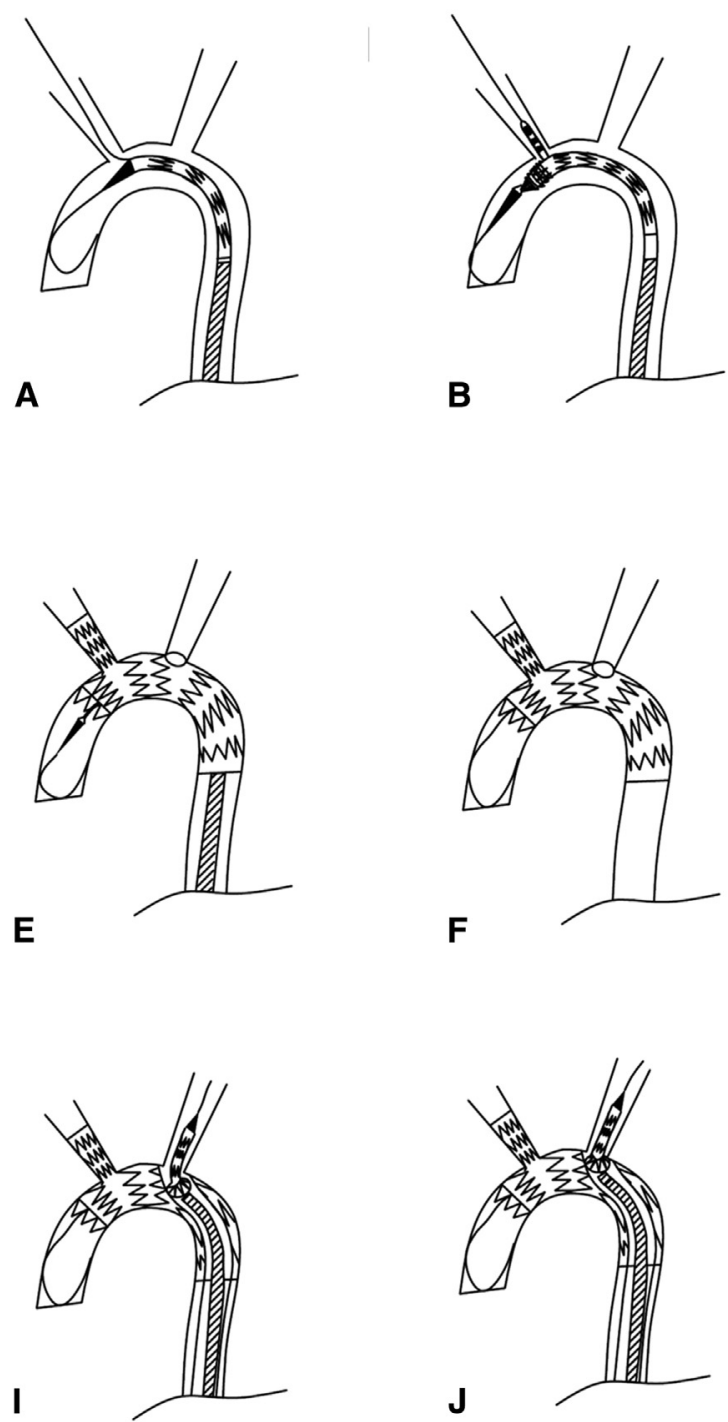

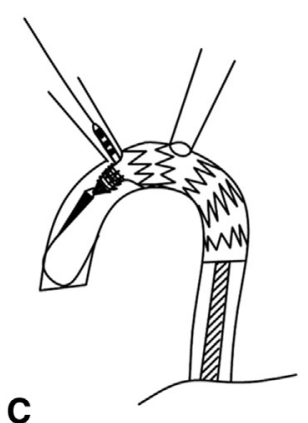

B
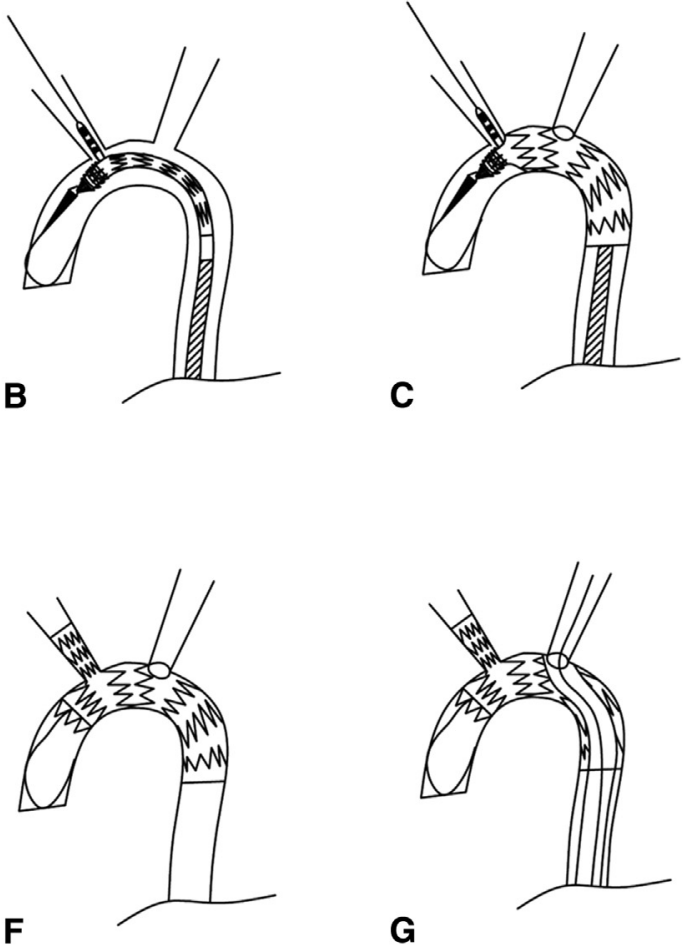

G

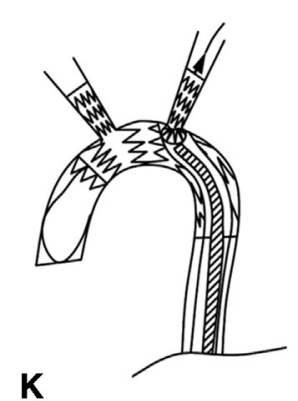

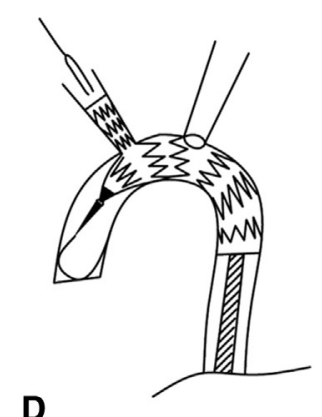

D

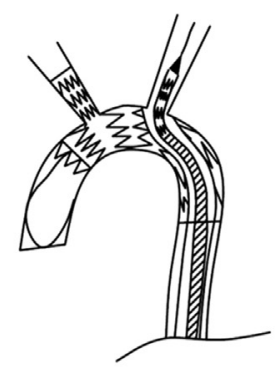

H

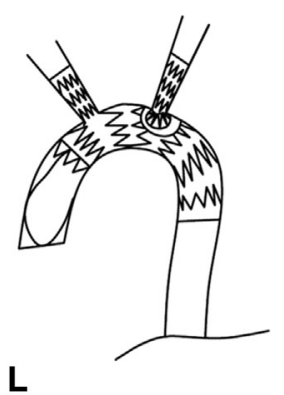

FIGURE 2. Schematic illustrations of the release procedure of Yanger stent-graft system. A, Both the delivery system and the compressed primary stent-graft were advanced into the aortic arch. B, The compressed branch was retracted into the BCT. C, The distal portion of the primary stent-graft was opened. D, The branch of the primary stent-graft was released. E, The front fixation of the primary stent-graft was opened. F, The delivery sheath of the primary stent-graft was retrieved. G, A super-slippery wire/stiff-wire was introduced into the LSCA. H, The delivery system of the derby hat branched stent-graft was advanced into the LSCA. I, The outer sheath was retrieved. J, The prebonded thread was retracted. K, The branched stent-graft was released. L, Total endovascular aortic arch reconstruction was complete.

of the primary stent-graft, ascending aorta angiography was performed to determine if an obstruction was present in the BCT or LSCA (Figure 4, $E)$. By using a multipurpose catheter, a super-slippery wire was introduced into the LSCA through a premade fenestration adjacent to the orifice of LSCA and exchanged for Lunderquist super-stiff wire (Figure 4, F). With the Lunderquist wire, the delivery system of the derby hat branched stent-graft was advanced into the LSCA (Figure 4, G). After retrieving the outer sheath and retracting the prebonded thread, the branched stent-graft was released (Figure 4, $H$ ). By retrieving the delivery system of the derby hat branched stent-graft, the procedure was complete. Repeat angiography was recommended to confirm both the position and the shape of the Yanger stent-graft system and to determine whether any valvular insufficiency, endoleak, brachiocephalic branch, or coronary artery obstruction was present (Figure 4,I). The whole angiography procedure of total endovascular stent-graft implantation into the aortic arch is presented in Video 1.

After stent-graft implantation, all experimental animals received postoperative intramuscular injections of penicillin and subcutaneous injections of low molecular weight heparin (2500 IU) for 3 days. The animals were bred for 6 months in an environment with a temperature of $15^{\circ} \mathrm{C}$ to $25^{\circ} \mathrm{C}$ and a humidity of $40 \%$, and received food and water 3 times daily. Oral aspirin ( $3 \mathrm{mg} / \mathrm{kg}$ of body weight) was administered for 30 consecutive days.

After successful stent-graft implantation, 2 animals were euthanized at 1 hour and 1 month, respectively, for stent-graft retrieval and histologic evaluation. The covered stent-graft was carefully removed from the aortic arch, and the surface of the stent-graft was examined via pathologic sectioning and scanning electron microscopy. 


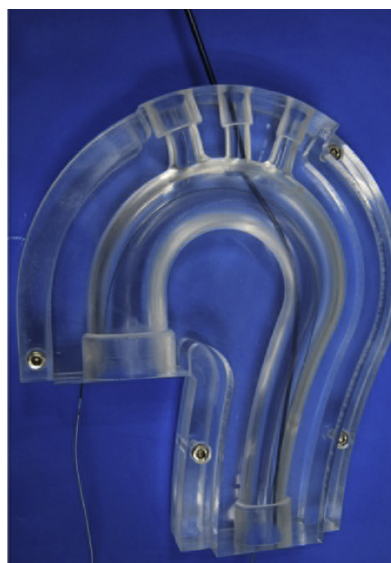

A

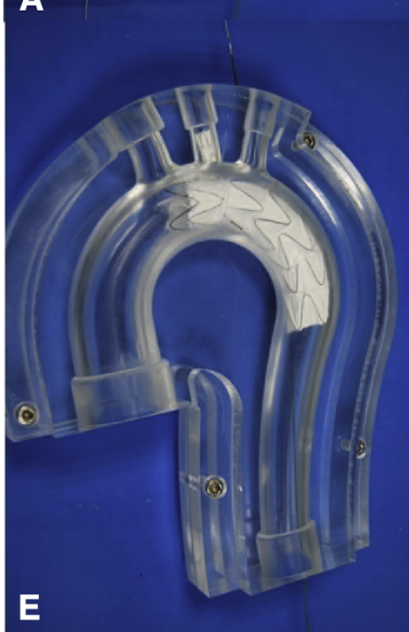

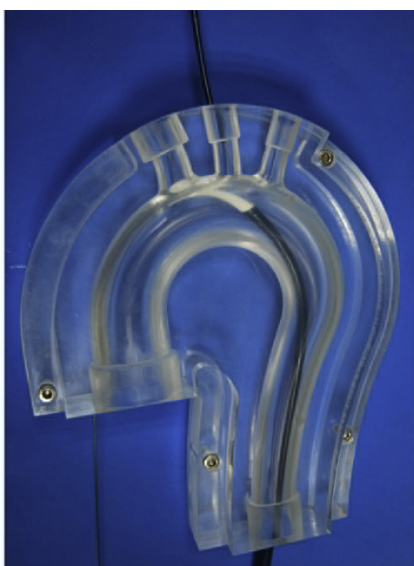

B

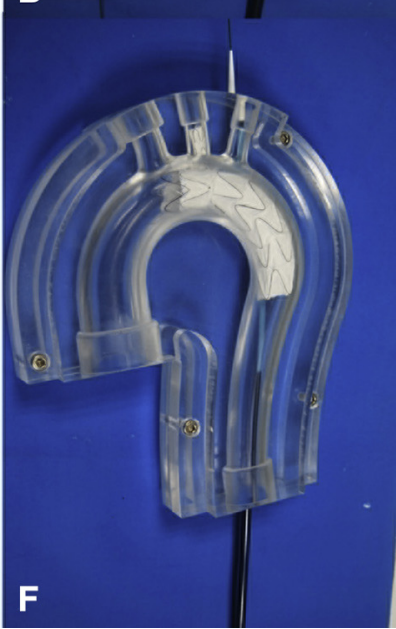

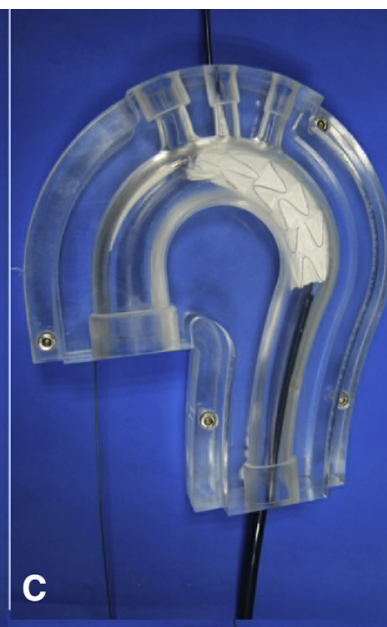
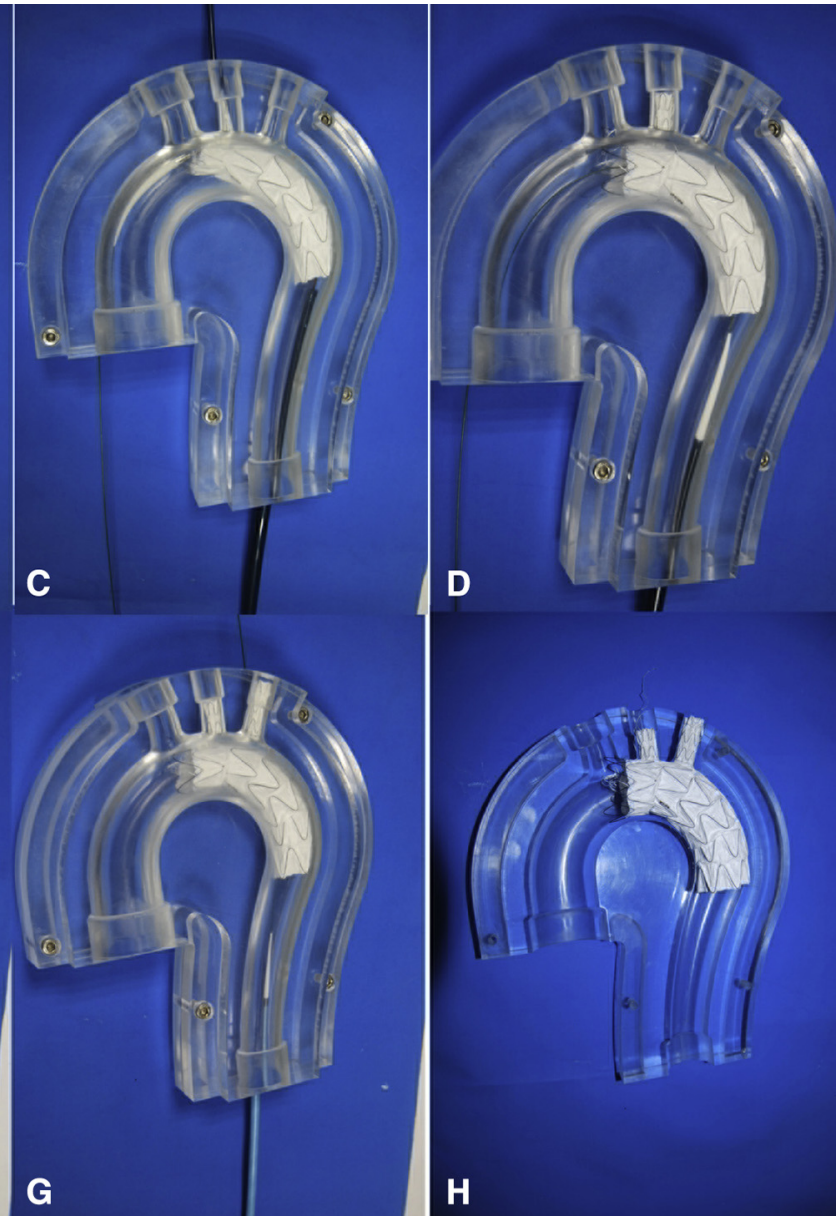

D

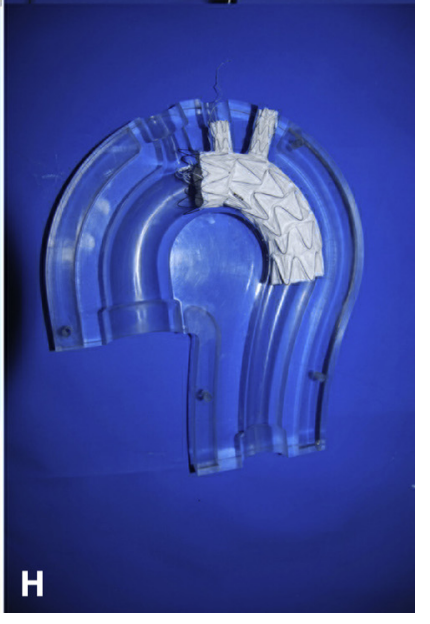

FIGURE 3. An in vitro test of the Yanger stent-graft system. A, Two wires were preloaded. B, The delivery system and compressed primary stent-graft were advanced into the aortic arch. C, The compressed branch was retracted into the BCT, and the distal portion of the stent-graft was opened. D, The branch of the primary stent-graft was released. E, A super-slippery wire/stiff-wire was introduced into the LSCA. F, The delivery system of the derby hat branched stent-graft was advanced into the LSCA. G, The branched stent-graft was released. H, Total endovascular aortic arch reconstruction was complete.

\section{Follow-up}

The dietary habits, health, mental status, and bowel activities of the animals were observed after surgery and during a 6-month follow-up period. At 6 months after stent-graft implantation, transthoracic color Doppler was used to evaluate the animals' ejection fractions, as well as the degree of aortic regurgitation, left ventricular volume change, degree of stenosis within any part of the graft, and blood flow velocity changes of each brachiocephalic artery. Multislice computed tomography (MSCT) was performed with appropriate contrast to assess the shape, location, and hemodynamic status of the stent-graft.

\section{Statistical Analysis}

Data were presented as means \pm standard deviations, medians with ranges, or frequencies or percentages, as appropriate. SPSS 16.0 for Windows (SPSS, Inc, Chicago, Ill) was used for the statistical analysis. The $t$ test, chi-square test, and Fisher exact test were used for comparisons between 2 groups, where appropriate.

\section{RESULTS}

\section{In Vitro Test Results}

Six sets of the Yanger stent-graft system were tested using an in vitro aortic arch simulation test bench, a test that was repeated 5 times (2 sets each of 24-20-160 mm, 28-24-160 mm, and 32-26-160 mm). A total of 30 in vitro tests were performed. Stent-graft release was successful during each of the 30 tests. However, 14 instances of branch wire entanglement with the Lunderquist super-stiff guidewire were noted. All entanglements were successfully resolved via clockwise rotation of the primary stent-graft delivery system. The branch delivery system was deployed through the fenestration during all tests, and the final position of each graft was satisfactory.

\section{Intraoperative Results and Clinical Follow-up}

Fourteen of the 16 animals underwent stent-graft implantation. Two deaths occurred periprocedurally. One animal died of peripheral vascular complications, and 1 animal died of an intraoperative arrhythmia when the external sheath was introduced into aortic arch. Ventricular fibrillation was most likely triggered via hemodynamic instability. Anatomic parameters were measured via angiography and 

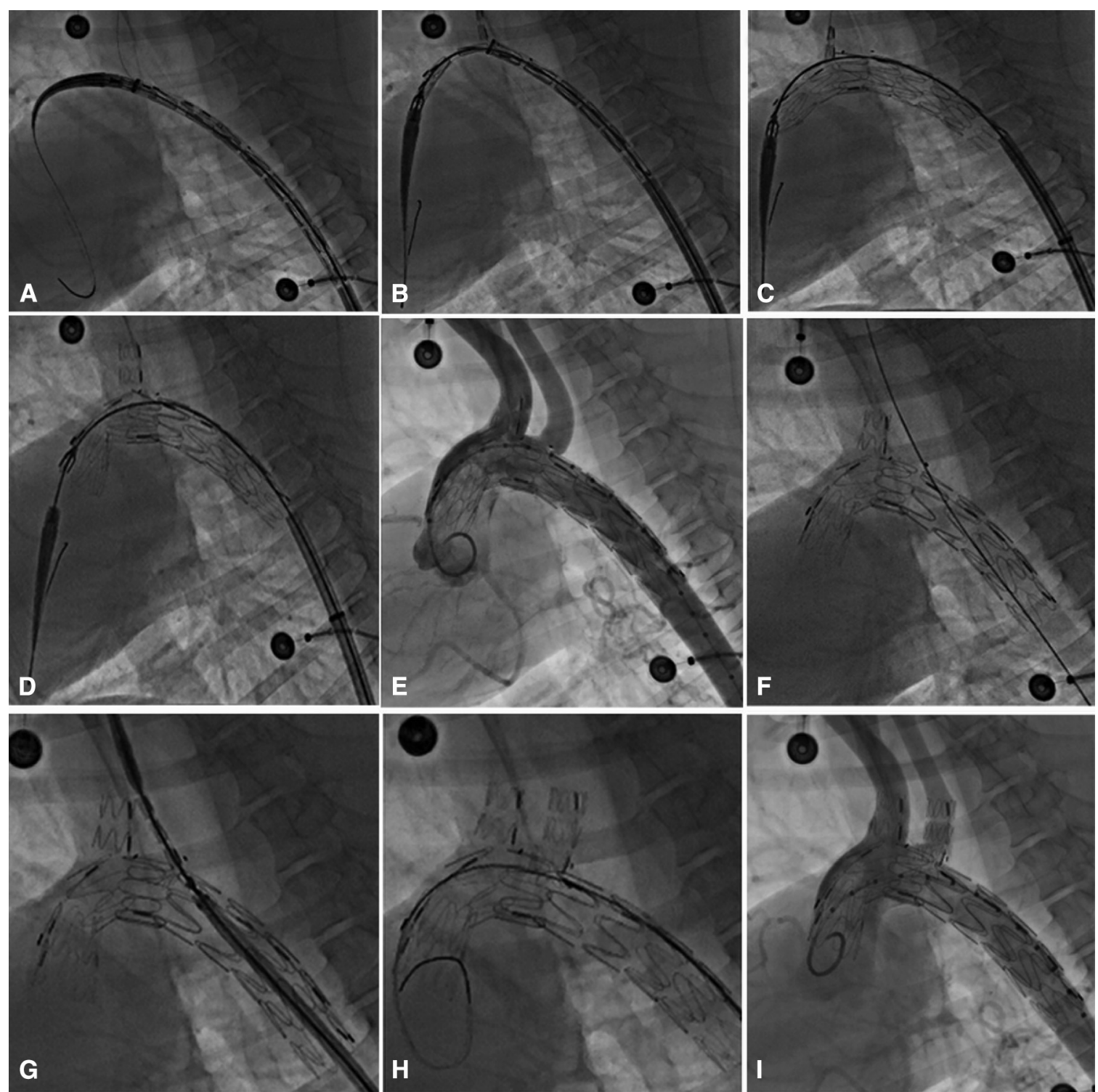

FIGURE 4. The release procedure of the Yanger stent-graft system in a canine model. A, The delivery system and compressed main stent-graft were advanced into the aortic arch. B, The compressed branch was retracted into the BCT. C, The distal portion of the primary stent-graft was opened. D, The branch of the primary stent-graft was released, and the front fixation of the primary stent-graft was opened. E, Ascending aortography demonstrated that the primary body of the Yanger stent-graft system was successfully released, without obstructing brachiocephalic branches. F, A stiff-wire was introduced into the LSCA. G, The delivery system of the derby hat branched stent-graft was advanced into the LSCA. H, After retrieving the outer sheath, retracting the prebonded thread, and retrieving the delivery system of the derby hat branched stent-graft, the procedure was complete. I, Repeat angiography demonstrated excellent positioning and shaping of the Yanger stent-graft system, as well as patent brachiocephalic branches and evidence of valvular insufficiency, endoleak, or coronary artery obstruction.

Doppler ultrasound. The mean diameter of the BCT was $11.7 \pm 1.1 \mathrm{~mm}$ via angiography and $11.4 \pm 1.2 \mathrm{~mm}$ via Doppler ultrasound. The diameters of the LSCA, ascending aorta, and descending aorta were $9.5 \pm 0.8 \mathrm{~mm}$, $25.3 \pm 2.0 \mathrm{~mm}$, and $18.9 \pm 1.3 \mathrm{~mm}$, respectively, via angiography. The distance between the BCT and the LSCA was $6.5 \pm 1.1 \mathrm{~mm}$, and the distance between the $\mathrm{BCT}$ and the annulus was $44.7 \pm 3.9 \mathrm{~mm}$. The mean $\mathrm{X}$-ray exposure time was $18.3 \pm 1.8$ minutes. The mean duration of the procedure was $101.7 \pm 13.1$ minutes (Table 1). No obvious endoleak was noted in all animals using angiography. Blood pressures, including systolic, diastolic, and mean pressures, were measured and recorded using a multipurpose catheter within the ascending aorta, descending aorta, distal to the BCT, and distal to the LSCA. No significant differences were noted (Figure 5). At 6 months of follow-up, no evidence of persistent atrioventricular block or ventricular arrhythmia was observed. 
TABLE 1. Baseline characteristics and procedural data of the animals that underwent endovascular aortic arch reconstruction (means \pm standard deviations)

\begin{tabular}{lc}
\hline Dogs $(\mathrm{n})$ & 16 \\
Sex $(\mathrm{F} / \mathrm{M})(\mathrm{n} / \%)$ & $7(43.8 \%) / 9(56.2 \%)$ \\
Age $(\mathrm{y})$ & $2.1 \pm 0.3$ \\
Weight $(\mathrm{kg})$ & $28.2 \pm 2.7$ \\
Diameter of BCT (mm) & $11.7 \pm 1.1$ \\
Distance between BCT and LSCA (mm) & $6.5 \pm 1.1$ \\
Diameter of LSCA (mm) & $9.5 \pm 0.8$ \\
Diameter of ascending aorta (mm) & $25.3 \pm 2.0$ \\
Diameter of descending aorta (mm) & $18.9 \pm 1.3$ \\
Distance between BCT and annulus (mm) & $44.7 \pm 3.9$ \\
X-ray exposure time (min) & $18.3 \pm 9.8$ \\
Operation time (min) & $101.7 \pm 13.1$ \\
\hline BCT, Bration
\end{tabular}

$B C T$, Brachiocephalic trunk; $L S C A$, left subclavian artery.

No significant occlusion or stenosis of the brachiocephalic branches was observed at 6 months of follow-up. There was no stent fracture or stent-graft migration by MSCT examination in all surviving animals at the 6-month follow-up. No paraplegia was found in our animal study series.

\section{Gross Anatomic and Histopathologic Examinations}

The macroscopic findings at autopsy demonstrated that the Yanger stent-graft was successfully deployed within the aorta; no crevices between the stent-graft and the native aorta were observed. At 1 month of follow-up, a macroscopic analysis demonstrated that the device was in the appropriate position and partially covered with endothelium. The position was consistent with the MSCT images. No macroscopic damage, including the erosion of atrial wall, was noted in the native aortas of individual animals.

\section{Multislice Computed Tomography and \\ Echocardiographic Evaluations}

The computed tomography angiography images demonstrated that the stent-grafts were in excellent position, with the proximal and distal portions located within the ascending and descending aortas, respectively. An axial scan demonstrated patent BCT and LSCA vasculature via stent-graft images of the arch, ascending aorta, and descending aorta (Figure 6).

Three-dimensional reconstruction MSCT images depicted the stent-graft at an appropriate position (Figure 7). The ascending aorta, descending aorta, BCT, and LSCA were each covered by the Yanger stent-graft system. There was no obstruction or stenosis noted in the brachiocephalic branches. No endoleak was observed between the stentgraft system and the native aortic wall. Echocardiographic imaging also demonstrated that the stent-grafts were in an appropriate position at 6 months of follow-up. MSCT after total endovascular stent-graft implantation into the aortic arch in canine models is shown in Videos 2-4.

\section{DISCUSSION}

Aneurysms and dissections involving the ascending aorta and arch historically have been treated through open

\section{preopretion}
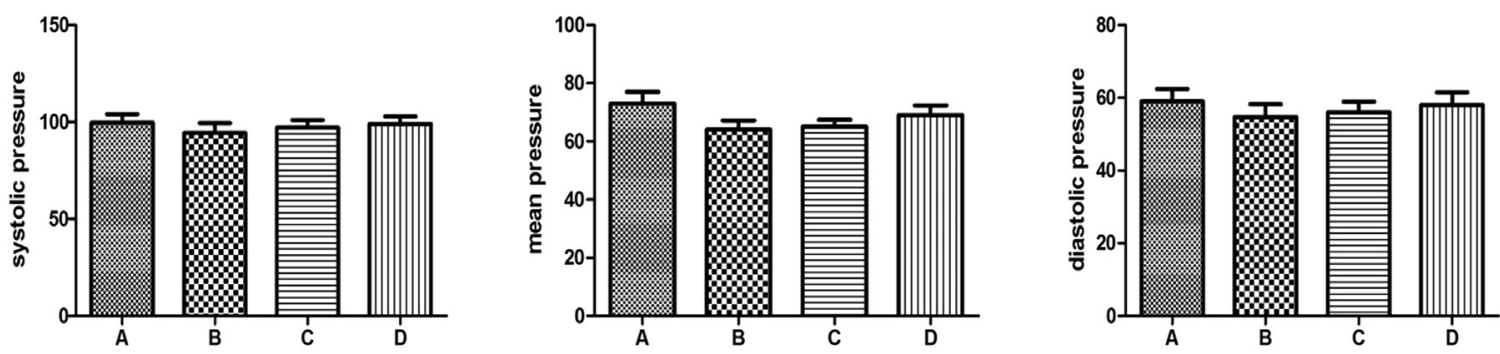

\section{postoperation}
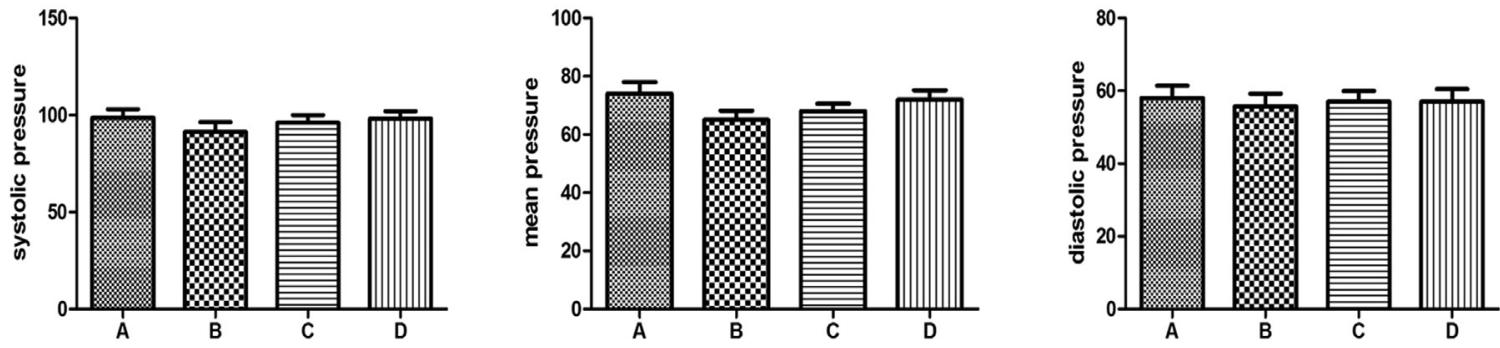

FIGURE 5. Blood pressure measurements at different positions pre- and postsurgery. A, Ascending aorta. B, Distal to the BCT. C, Distal to the LSCA. $\mathrm{D}$, Descending aorta. 


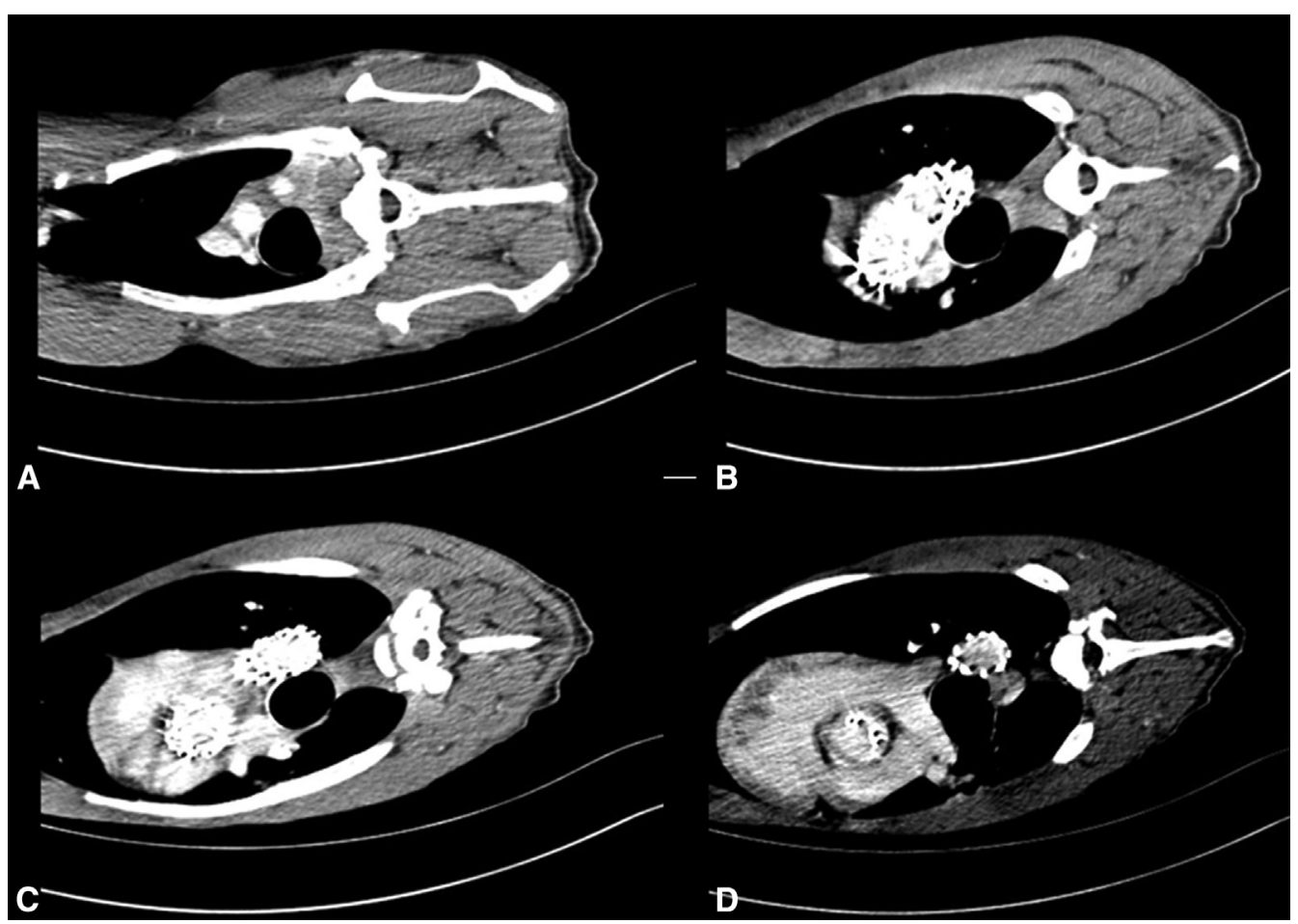

FIGURE 6. Axial MSCT images after the deployment of the Yanger stent-graft system. A, Brachiocephalic branch level. B, Arch level. C, Descending aortic level. D, Sinotubular junction level.

surgical techniques requiring cardiopulmonary bypass and under deep hypothermic circulatory arrest. ${ }^{11,14,15}$ Despite continued refinements to these procedures, these procedures are still associated with substantial mortality and morbidity, particularly among the elderly. ${ }^{16}$ Since the invention of the endovascular procedure by Parodi ${ }^{17}$ to treat aortic aneurysms, both endovascular abdominal aneurysm repair and TEVAR have gained recognition as alternatives to conventional surgical repair in the treatment of various aortic morphologies. ${ }^{18,19}$ Although it is not the standard care for retrograde dissection and arch pathology, TEVAR and other surgical procedures as debranching techniques have been used to treat retrograde extension into the arch and ascending aorta, because coverage of the entry site is needed to induce aortic remodeling and healing. ${ }^{20-22}$ However, these hybrid approaches are not recommended as the first option in the treatment of aortic arch lesions.

Experience gained from endovascular abdominal aneurysm repair and recent advances in branch and fenestrated stent-grafts in the treatment of abdominal aortic aneurysms have made the treatment of more complex anatomy possible. ${ }^{23-25}$ Fenestrated endografts, the chimney technique, and in situ fenestration also have become options for endovascular aortic arch repair. ${ }^{26-29}$ Among these techniques, the endograft manufactured by Cook Inc seems to be the most promising. Preliminary human studies have confirmed both the short-term safety and the effectiveness of this system. ${ }^{28,29}$ However, this system and other fenestrated/branched stent-grafts do not allow for anatomic adaption to the native curvature of the arch and brachiocephalic vessels.

On the basis of the previous design of the branched stent-graft, as well as other techniques, ${ }^{30,31}$ we designed a new stent-graft system with the assistance of the research and engineering staff of LifeTech Scientific. The Yanger stent-graft system is designed to fully adapt to the native anatomy of the aortic arch, which contrasts with previous designs. The in vitro tests performed during our study demonstrated the effectiveness of the system's release.

We also demonstrated the technical feasibility of endovascular treatment of the aortic arch using a simplified branched stent-graft in a canine model. The Yanger stent-graft system was implanted with each of the supra-arch branches preserved and protected. On the basis of our experience, to achieve satisfactory results, several crucial steps should be taken into consideration. First and foremost, avoiding entanglements between the branch wire and the Lunderquist super-stiff guidewire is essential. Clockwise rotation of the primary stent-graft delivery system allows for resolution of the entanglement, both in vitro and in an animal model. Second, femoral arteries of dogs are relatively small and do not represent good entry points for the stent-graft system. Laparotomy and the abdominal artery are recommended as entry points to avoid 


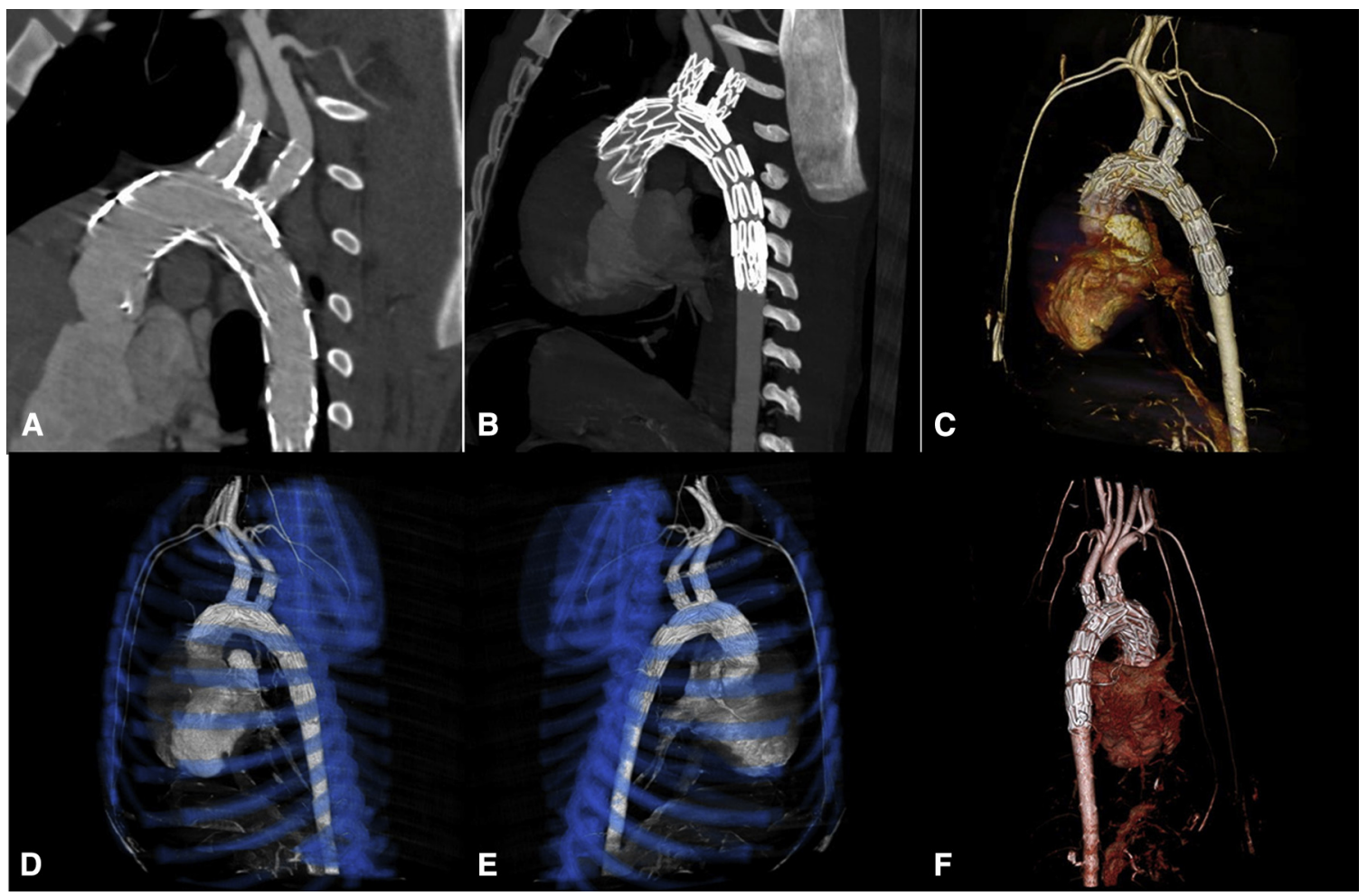

FIGURE 7. Three-dimensional reconstruction MSCT images after the deployment of the Yanger stent-graft system at 6 months of follow-up. A, Sagittal view of $\operatorname{dog} 3$. B, Reconstruction image of $\operatorname{dog} 3$. C, Reconstruction image of $\operatorname{dog} 9$. D, Reconstruction image of dog 9 , frontal view. E, Reconstruction image of $\operatorname{dog} 9$, rear view. F, Reconstruction image of $\operatorname{dog} 15$.

peripheral vessel complications. Third, the sharp angulation of the canine BCT made it difficult to insert the cannula. Simultaneous stretching of both ends of the guidewire may help the catheters advance through the BCT. The prevention of embolic cerebrovascular accidents is an additional consideration. The release of the branch stent-graft of the BCT and LSCA was performed via the transfemoral retrograde approach in our study. No sheath was necessary to enter the BCT and LSCA, which significantly reduced the incidence of embolic cerebrovascular events. In our animal experiments, our stent-graft implantation technique appeared to be safe with respects to the risk of an embolic cerebrovascular event.

\section{Study Limitations}

Our preclinical study suffered from several limitations. First and foremost, the canine model offers only a partial simulation of native human anatomy. The anatomic variations found among humans are far more complex than those in canine models. Second, the canine model is a physiologic model and does not allow for the mimicry of dissection or aneurysm. Third, the long-term durability of the branched stent-grafts in aortic arch is unknown. Therefore, long-term follow-up is necessary to address this issue. Finally, the devices were not individualized. Thus, the stent-graft may be too small or too large.

\section{CONCLUSIONS}

With the use of a canine model, our study demonstrated both the safety and the efficacy of the Yanger stent-graft system, which has been successfully implanted in an appropriate and stable position, and patent brachiocephalic flow was also demonstrated. The ascending aorta, aortic arch, BCT, LSCA, and descending aorta were each protected by the endograft. The application of this graft system for the total endovascular repair of the aortic arch is both feasible and reliable. Theoretically, the application of this technique may be expanded to a wide range of therapies involving the ascending aorta and the aortic arch using individualized devices. The ethics committee of Xijing Hospital has approved the device for limited clinical use (KY20141017-1); this trial is registered with ClinicalTrials.gov (NCT01496833 TEARS study). With increasing preclinical knowledge, both patient selection criteria and the first in-human studies will be addressed.

\section{Conflict of Interest Statement}

J.Y. receives institutional research grants and consulting fees from Lifetech Scientific, Shenzhen, China. The Xijing Hospital holds the patent rights to the device, and Lifetech Scientific provided the stent-grafts used in the study. All other authors have nothing to disclose with regard to commercial support. 
The authors thank Gang Wang, PhD, Mingyang Cai, PhD, and the entire research staff, led by Deyuan Zhang, $\mathrm{PhD}$, of LifeTech Scientific, Shenzhen, China.

\section{References}

1. Ince $\mathrm{H}$, Nienaber CA. Etiology, pathogenesis and management of thoracic aortic aneurysm. Nat Clin Pract Cardiovasc Med. 2007;4:418-27.

2. Ishimaru S. Endografting of the aortic arch. J Endovasc Ther. 2004;11(Suppl 2): II62-71.

3. Weigang E, Parker J, Czerny M, Peivandi AA, Dorweiler B, Beyersdorf F, et al. Endovascular aortic arch repair after aortic arch de-branching. Ann Thorac Surg. 2009;87:603-7.

4. Sun L, Qi R, Zhu J, Liu Y, Zheng J. Total arch replacement combined with stented elephant trunk implantation: a new "standard" therapy for type a dissection involving repair of the aortic arch? Circulation. 2011; 123:971-8.

5. Nienaber CA, Fattori R, Lund G, Dieckmann C, Wolf W, von Kodolitsch Y, et al. Nonsurgical reconstruction of thoracic aortic dissection by stent-graft placement. N Engl J Med. 1999;340:1539-45.

6. Di Bartolomeo R, Pantaleo A, Berretta P, Murana G, Castrovinci S, Cefarelli M, et al. Frozen elephant trunk surgery in acute aortic dissection. J Thorac Cardiovasc Surg. 2015;149:S105-9.

7. Roselli EE, Rafael A, Soltesz EG, Canale L, Lytle BW. Simplified frozen elephant trunk repair for acute DeBakey type I dissection. J Thorac Cardiovasc Surg. 2013;145:S197-201.

8. Greenberg RK, Haddad F, Svensson L, O’Neill S, Walker E, Lyden SP, et al. Hybrid approaches to thoracic aortic aneurysms: the role of endovascular. Circulation. 2005;112:2619-26.

9. Verhoye JP, Anselmi A, Kaladji A, Flecher E, Lucas A, Heautot JF, et al. Mid-term results of elective repair of extensive thoracic aortic pathology by the Evita Open Plus hybrid endoprosthesis only. Eur J Cardiothorac Surg. 2014;45:812-7.

10. Ius F, Hagl C, Haverich A, Pichlmaier M. Elephant trunk procedure 27 years after Borst: what remains and what is new? Eur J Cardiothorac Surg. 2011;40: $1-11$.

11. Tang GH, Kai M, Malekan R, Lansman SL, Spielvogel D. Trifurcated graft replacement of the aortic arch: state of the art. J Thorac Cardiovasc Surg. 2015;149:S55-8.

12. Inoue K, Hosokawa H, Iwase T, Sato M, Yoshida Y, Ueno K, et al. Aortic arch reconstruction by transluminally placed endovascular branched stent graft. Circulation. 1999;100:II316-21.

13. Chuter TA, Hiramoto JS, Chang C, Wakil L, Schneider DB, Rapp JH, et al. Branched stent-grafts: will these become the new standard? J Vasc Interv Radiol. 2008;19:S57-62.

14. Nienaber CA, Eagle KA. Aortic dissection: new frontiers in diagnosis and management: Part I: from etiology to diagnostic strategies. Circulation. 2003; 108:628-35.

15. Nienaber CA, Eagle KA. Aortic dissection: new frontiers in diagnosis and management: Part II: therapeutic management and follow-up. Circulation. 2003;108:772-8.
16. Moon MC, Morales JP, Greenberg RK. The aortic arch and ascending aorta: are they within the endovascular realm? Semin Vasc Surg. 2007;20:97-107.

17. Parodi JC. Endovascular stent graft repair of aortic aneurysms. Curr Opin Cardiol. 1997;12:396-405.

18. Dake MD, Kato N, Mitchell RS, Semba CP, Razavi MK, Shimono T, et al. Endovascular stent-graft placement for the treatment of acute aortic dissection. N Engl J Med. 1999;340:1546-52.

19. Fattori R, Cao P, De Rango P, Czerny M, Evangelista A, Nienaber C, et al. Interdisciplinary expert consensus document on management of type $\mathrm{b}$ aortic dissection. J Am Coll Cardiol. 2013;61:1661-78.

20. Greenberg RK, Haddad F, Svensson L, O’Neill S, Walker E, Lyden SP, et al. Hybrid approaches to thoracic aortic aneurysms: the role of endovascular elephant trunk completion. Circulation. 2005;112:2619-26.

21. Nienaber CA, Clough RE. Management of acute aortic dissection. Lancet. 2015; 385:800-11.

22. Hiraoka A, Chikazawa G, Tamura K, Totsugawa T, Sakaguchi T, Yoshitaka H. Clinical outcomes of different approaches to aortic arch disease. J Vasc Surg. 2015;61:88-95.

23. Greenberg RK, Lytle B. Endovascular repair of thoracoabdominal aneurysms. Circulation. 2008;117:2288-96.

24. Greenberg R, Eagleton M, Mastracci T. Branched endografts for thoracoabdominal aneurysms. J Thorac Cardiovasc Surg. 2010;140:S171-8.

25. O'Brien N, Sobocinski J, d'Elia P, Guillou M, Maioli F, Azzaoui R, et al. Fenestrated endovascular repair of type IV thoracoabdominal aneurysms: device design and implantation technique. Perspect Vasc Surg Endovasc Ther. 2011;23: 173-7.

26. Malina M, Sonesson B. In situ fenestration: a novel option for endovascular aortic arch repair. J Thorac Cardiovasc Surg. 2015;56:355-62.

27. Kasemi H, Marino M, Di Angelo CL, Fadda GF, Speziale F. Aortic arch and descending thoracic aortic saccular aneurysms treatment with fenestrated endograft and chimney technique for aortic branch rescue. Ann Vasc Surg. 2015;29:126.e15-9.

28. Tse LW, Lindsay TF, Roche-Nagle G, Oreopoulos GD, Ouzounian M, Tan KT. Radiofrequency in situ fenestration for aortic arch vessels during thoracic endovascular repair. J Endovasc Ther. 2015;22:116-21.

29. Kurimoto Y, Maruyama R, Ujihira K, Nishioka N, Hasegawa K, Iba Y, et al. Thoracic endovascular aortic repair for challenging aortic arch diseases using fenestrated stent grafts from zone 0. Ann Thorac Surg. 2015;100:24-32.

30. Lin C, Lu Q, Liao M, Guo M, Gong J, Jing Z. Endovascular repair of the half aortic arch in pigs with an improved, single-branched stent graft system for the brachiocephalic trunk. Vascular. 2011;19:242-9.

31. Lin C, Wang L, Lu Q, Li C, Jing Z. Endovascular repair of the aortic arch in pigs by improved double-branched stent grafts. Ann R Coll Surg Engl. 2013; 95:134-9.

Key Words: thoracic endovascular aortic repair (TEVAR), deep hypothermic circulatory arrest (DHCA), brachiocephalic trunk (BCT), left subclavian artery (LSCA), multisliced CT (MSCT) 